\title{
Sistematización de experiencias en el fortalecimiento de capacidades de Acueductos Comunales (Asadas) en Santa Cruz, Abangares, La Cruz y Nicoya
}

\section{Systematization of Experiences in Capacity Building of Community Aqueducts (ASADAS) in Santa Cruz, Abangares, La Cruz, and Nicoya, Costa Rica}

Álvaro Baldioceda Garro

Universidad Nacional

Liberia, Costa Rica alvaro.baldioceda.garro@una.ac.cr

Anny Guillén Watson

Universidad Nacional Liberia, Costa Rica anny.guillen.watson@una.ac.cr

Johanna Rojas Conejo

Universidad Nacional Liberia, Costa Rica johanna.rojas.conejo@una.ac.cr

Andrea Suárez Serrano

Universidad Nacional

Liberia, Costa Rica andrea.suarez.serrano@una.ac.cr

Christian Golcher Benavides

Universidad Nacional Liberia, Costa Rica christian.golcher.benavides@una.ac.cr

William Gómez Solis

Universidad Nacional Liberia, Costa Rica william.gomez.solis@una.ac.cr

Recibido: 13/04/2020 Aceptado: 07/08/2020 
Resumen. El objetivo de este artículo es presentar información acerca de la sistematización de experiencias del proyecto: "Fortalecimiento de las capacidades de las Asociaciones Administradoras de Acueductos Rurales (Asadas) mediante la capacitación e implementación de mejores prácticas, técnicas e innovación en la gestión comunitaria del agua", con la idea de compartir los aprendizajes adquiridos por las personas proyectistas y los principales hitos encontrados durante este proceso. El proyecto tuvo una duración de cuatro años e incluyó a treinta y dos Asadas de la región Chorotega. Los programas de capacitación y análisis se llevaron a cabo mediante el acompañamiento profesional, lo que permitió identificar las áreas vulnerables de estas organizaciones y trazar agendas de trabajo para solventar las problemáticas de manera individualizada. Se pudo observar que existe una marcada diferencia a nivel económico, de infraestructura y administrativa de los distintos acueductos participantes, por lo que las metodologías de trabajo deben ser ajustadas para cada Asada. Además, los procesos de acompañamiento son exitosos siempre y cuando se desarrollen relaciones de confianza entre las Asadas y la universidad.

Palabras clave: gestión comunitaria del agua, cantidad de agua, vulnerabilidad, empoderamiento y sistematización.

Abstract. This article describes the systematization process of the project titled "Enhancing capacities of Rural Aqueduct Associations (ASADAS) by implementing good practices, techniques, and innovation in community water management." The article shares the different experiences lived by the extensionists and the significant highlights observed through the process. The project lasted four years and involved 32 community water operators from the Chorotega Region (Costa Rica's North Pacific region), named ASADAS. Capacity -building processes were individually put in place to reduce vulnerability and define incoming goals addressing specific problems. We could observe differences between the participant water operators at the economic, infrastructure, and administrative levels. Therefore, interventions were adjusted for each ASADA, strengthening the relationship between these water stakeholders and the university.

Keywords: community water management and empowerment, water quality and quantity, vulnerability.

\section{Introducción}

En Costa Rica existen diferentes operadores que se encargan de la gestión del agua en términos de la administración y distribución. Se destacan el Instituto Costarricense de Acueductos y Alcantarillados (AyA) -ente rector nacional-, la Empresa de Servicios Públicos de Heredia (ESPH), las municipalidades 
URL: https://www.revistas.una.ac.cr/index.php/dialogo/index

CORREO ELECTRÓNICO: universidadendialogo@una.ac.cr

DOI: https://doi.org/10.15359/udre.10-2.1

y las Asociaciones Administradoras de Acueductos Comunales, llamadas Asadas (Acueductos y Alcantarillados, 2010).

Las Asadas se encargan del manejo de aproximadamente 1890 acueductos en todo el país. En términos de la población de Costa Rica, atienden cerca del $30 \%$ del total (Instituto Costarricense de Acueductos y Alcantarillados, 2016). Estas asociaciones son grupos de personas que se organizan en una comunidad para crear y administrar la infraestructura, las nacientes y los pozos, a fin de proveer el servicio de abastecimiento seguro de agua a la comunidad. El AyA le confiere a las Asadas un convenio de delegación con el cual se comprometen a velar por la captación, el tratamiento, la potabilización, la distribución y el mantenimiento de la red de tuberías (Estado de la Nación, 2007).

Guanacaste es una de las provincias de Costa Rica que más depende de las Asadas para lograr la distribución de agua potable a la población. Son aproximadamente 327 acueductos (Acueductos y Alcantarillados, 2010) que comparten condiciones que los hacen altamente vulnerables; el aumento de la infraestructura residencial y comercial por el crecimiento de la actividad turística, la disminución de caudales superficiales y subterráneos y la salinización de pozos en acueductos costeros. Por causa de estas problemáticas se generan las sequías y la sobreexplotación de las fuentes (Van Noorloos, 2013).

Se ha comprobado que las inversiones preventivas en sistemas de abastecimiento de agua de calidad y de saneamiento pueden ser rentables desde un punto de vista económico, ya que la disminución de los efectos adversos para la salud y la consiguiente reducción de los costos derivados de las externalidades ambientales negativas son superiores al costo de las intervenciones (OMS, 2004).

Por lo tanto, durante el año 2015 y el 2018, el proyecto Conare de Regionalización (CRI) desarrollado por el Centro de Recursos Hídricos para Centroamérica y el Caribe de la Universidad Nacional (Hidrocec-UNA) se planteó como objetivo fortalecer las capacidades de estas asociaciones mediante la capacitación e implementación de mejores prácticas, técnicas e innovación en la gestión comunitaria del agua. Esto promueve la adaptación y la resiliencia de las poblaciones de la región y de sus habitantes ante la vulnerabilidad y las amenazas, en particular de desastres climáticos e hidrometeorológicos.

El desarrollo de este proyecto ha permitido, a través del diálogo conjunto en talleres y otras actividades, una relación universidad-comunidad muy importante en la sostenibilidad de los objetivos de desarrollo. Esta experiencia 
ha revelado las múltiples problemáticas que padecen tanto las Asadas como las comunidades a las que pertenecen.

Para estas problemáticas pueden generarse hojas de ruta para la búsqueda de soluciones endógenas, soluciones desarrolladas desde la población. No obstante, es muy importante que no radiquen en la "simple" capacitación de los actores, pues el seguimiento y el acompañamiento técnico son actividades de largo plazo, como objetivos de desarrollo que son.

Los esfuerzos metodológicos de sistematización de estas intentan, por medio de la descripción de acontecimientos, descubrir los hitos que marcaron cada una de las actividades desarrolladas en el proyecto. Esto podría permitir, por ejemplo, ofrecer lecciones aprendidas en estas zonas del país, favoreciendo los procesos, minimizando los retos y maximizando las rutas y los objetivos.

En este caso, se utilizó el manual para sistematizar experiencias de manejo participativo (referencia) con el objetivo de dar a conocer cómo se realizaron los procesos socioparticipativos, y cómo estos han fortalecido el empoderamiento de las comunidades, la construcción de agendas de seguimiento y la materialización de proyectos desarrollados desde la población.

\section{Ubicación geográfica}

El estudio se ubica en la provincia de Guanacaste, propiamente en los cantones de Santa Cruz, Abangares, La Cruz y Nicoya. Específicamente, se trabajaron las siguientes Asadas:

- Santa Cruz: El Llano, Playa Potrero, Villarreal, Santa Rosa, Tamarindo, Huacas, San José de Pinilla, Matapalo, Playa Brasilito, La Garita, Lorena, Hernández y Cebadilla

- Abangares: Santa Lucía, Coyolar, Acueducto Municipal, San Juan Grande y Lourdes

- La Cruz: Santa Cecilia, La Garita, San Dimas, Las Brisas, La Amistad, Colonia Bolaños y Cuajiniquil

- Nicoya: Playa Sámara, El Piave Matamba, Dulce Nombre, San Antonio, Caimital, La Virginia, Gamalotal, Las Y Griegas y Santa Ana en el cantón de Nicoya 
URL: https://www.revistas.una.ac.cr/index.php/dialogo/index

CORREO ELECTRÓNICO: universidadendialogo@una.ac.cr

DOI: https://doi.org/10.15359/udre.10-2.1

Figura 1. Ubicación geográfica y administrativa de las Asadas.

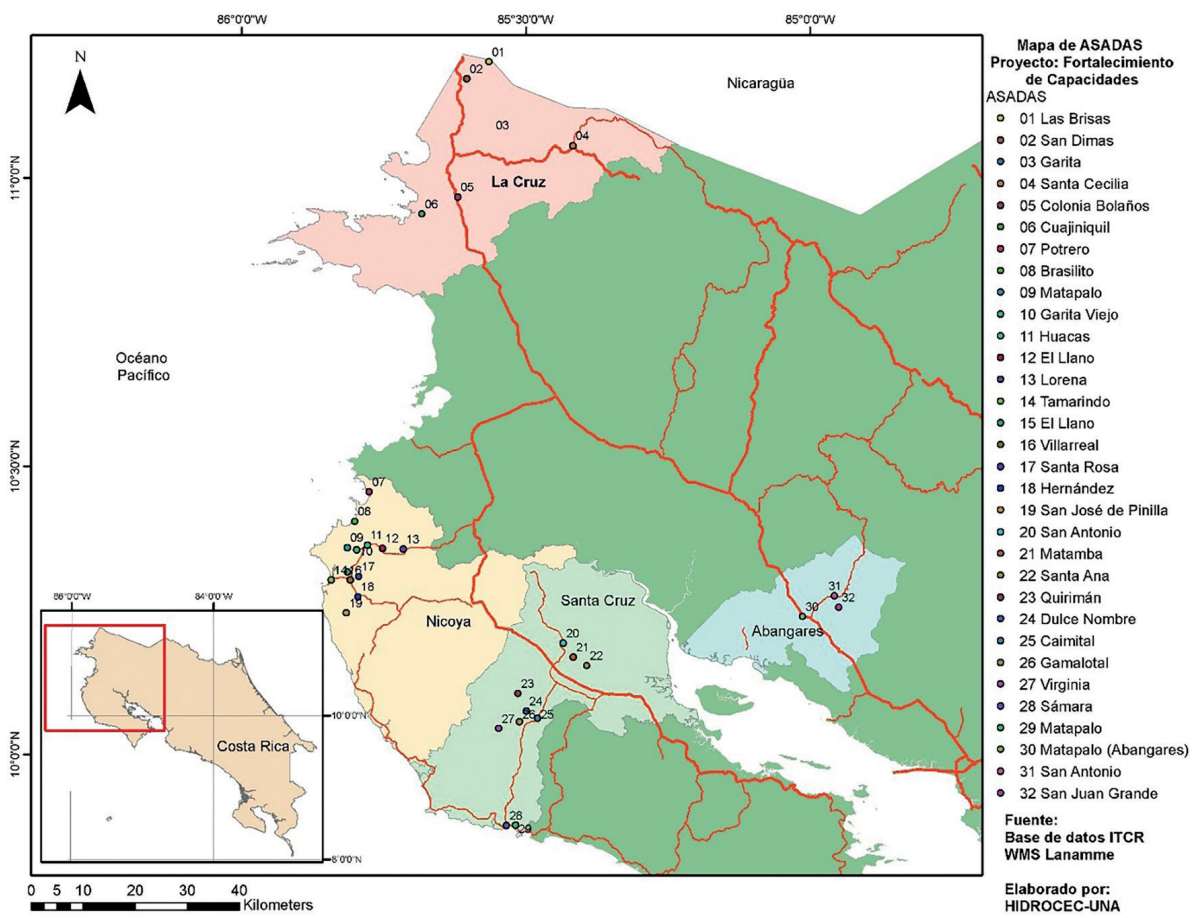

Fuente: Elaboración propia del HIDROCEC-UNA con base de datos de ITCR y WMS Lanamme.

\section{Metodología}

\section{Selección del grupo de Asadas participantes}

En el inicio del proceso se realizaron tres reuniones con las personas proyectistas del equipo de trabajo interdisciplinario. Se elaboró la línea base de información existente mediante investigación bibliográfica y de campo, con visitas a los actores relacionados con la gestión hídrica (AyA, ORAC, municipalidades, Ministerio de Salud). Se analizaron los instrumentos de aplicación realizados a las Asadas por diferentes instituciones para el diagnóstico de las condiciones actuales de cada Asada y la posibilidad de participación en el proyecto. Entre 
Revista Universidad en Diálogo • Vol. 10, N. 2, Julio-Diciembre, 2020 • 11-33

ISSN 2215-2849 • EISSN: 2215-4752

URL: https://www.revistas.una.ac.cr/index.php/dialogo/index CorReo eleCtrónICo: universidadendialogo@una.ac.cr DOI: https://doi.org/10.15359/udre.10-2.1

los principales productos ejecutados en esta primera fase se tiene una lista de las Asadas potencialmente participantes, las memorias y minutas de las reuniones, la consolidación de los grupos de trabajo y el diagnóstico con las condiciones actuales de algunas Asadas de la región Chorotega.

Inicialmente, el proyecto en alianza con la Subgerencia de Sistemas Comunales del AyA y las municipalidades de la zona se identificaron las Asadas más afectadas por las condiciones de sequía, escasez o salinización en el 2014 y el 2015 , con el fin de impulsar sus capacidades de emprender procesos de identificación y prevención de riesgos de la calidad de los recursos hídricos superficiales y subterráneos. Para el 2015, el proyecto identificó dos áreas geográficas prioritarias: la zona costera de Santa Cruz y el cantón de Abangares. Para los años 2016 y 2017, se identificó a Nicoya como otra área prioritaria, mientras que el cantón de La Cruz fue seleccionado en el 2018.

Una vez identificados los sitios de interés, se realizó el proceso de selección de cada una de las Asadas mediante la firma de acuerdos voluntarios de participación y la firma de consentimientos informados. En Santa Cruz el proceso arrancó con trece Asadas, a saber: El Llano, Playa Potrero, Villarreal, Santa Rosa, Tamarindo, Huacas, San José de Pinilla, Matapalo, Playa Brasilito, La Garita, Lorena, Hernández y Cebadilla; y para el cantón de Abangares con cinco: Santa Lucía, Coyolar, Acueducto Municipal, San Juan Grande y Lourdes. En 2016 y 2017 el proyecto identificó las Asadas del cantón de Nicoya como prioritarias, de las cuales se seleccionaron nueve: Playa Sámara, El Piave Matamba, Dulce Nombre, San Antonio, Caimital, La Virginia, Gamalotal, Las Y Griegas y Santa Ana. Mientras que en el 2018 se trabajaron siete Asadas de La Cruz: Cuajiniquil, Colonia Bolaños, Las Brisas, La Garita, San Dimas, La Amistad y Santa Cecilia.

\section{Trabajo socioparticipativo}

Los abordajes socioparticipativos no se enfocan en los individuos, sino en un conjunto de personas involucradas, directa o indirectamente, en el trabajo del cual son parte. La participación implica un trabajo de gestión concertada entre varios actores necesarios, entre beneficiarios directos, organismos gubernamentales y no gubernamentales, en operaciones de mejoramiento de la situación de la Asada, utilizando los recursos y las potencialidades de cada uno.

Valorando lo anterior, para iniciar este proceso se aplicó a cada una de las Asadas el "Formulario unificado del Instituto Costarricense de Acueductos y 
URL: https://www.revistas.una.ac.cr/index.php/dialogo/index

CORREO ELECTRÓNICO: universidadendialogo@una.ac.cr

DOI: https://doi.org/10.15359/udre.10-2.1

Alcantarillados Subgerencia Gestión de Sistemas Comunales" (AyA, 2015). Este instrumento fue desarrollado por diversas instituciones, entre las cuales están la Universidad Nacional, la Universidad de Costa Rica, la Universidad Técnica Nacional, el Ministerio de Salud, el Servicio Nacional de Riego y Avenamiento (Senara), la Autoridad Reguladora de los Servicios Públicos (Aresep) y el Ministerio de Ambiente y Energía (Minae).

La aplicación de este instrumento brinda insumos necesarios y comparables para la evaluación de cada una de las asociaciones, conociendo sus fortalezas y sus debilidades. Posteriormente, se hizo un análisis comparativo entre los casos, donde se detectaron diferencias y semejanzas entre los entes operadores, con el fin de construir la temática de los módulos de capacitación que influencien de mejor manera a cada Asada.

El trabajo socioparticipativo se realizó a través del desarrollo de talleres de trabajo con las Asadas, con el fin de plantear mejoras en las capacidades de los acueductos y la prestación del servicio de agua potable. Se adaptó la metodología propuesta por la Organización Mundial de la Salud de Planes de Seguridad del Agua a las particularidades de las comunidades. En total, se desarrollaron ocho módulos de trabajo con una duración de doce semanas en sesiones de cuatro horas semanales. Los módulos desarrollados fueron: 1) Gestión Integrada del Recurso Hídrico (GIRH), 2) Calidad de Aguas, 3) Medición, Ahorro y Uso Eficiente del Agua, 4) Manejo Administrativo de Asadas, 5) Introducción a los Planes de Seguridad del Agua, 6) Mantenimientos Operativos, 7) Gestión de Riesgo y 8) Llegando a Acuerdos.

El desarrollo de los módulos estuvo a cargo del grupo interdisciplinario compuesto por académicos de la Sede Regional Chorotega y de estudiantes avanzados de Administración de la Universidad Nacional, quienes fueron seleccionados por la coordinación de su respectiva carrera. Durante su aplicación, se buscó la construcción integral del conocimiento, por lo que se combina la exposición teórica con actividades prácticas y con la aplicación de los conceptos. Esto mediante una constante discusión con apoyo de herramientas tecnológicas.

Paralelamente, todas las Asadas participantes fueron evaluadas mediante campañas de muestreo, según lo establecido en el Reglamento de Calidad de Agua Potable No 38924-S (Presidencia de la República, Ministerio de Salud, 2015). En este reglamento se indica el número de puntos de muestreo según la población abastecida por la misma. En cuanto a las Asadas involucradas en el estudio, era necesario tomar puntos en la fuente, en el tanque de 
almacenamiento y en tres puntos a lo largo de la red de distribución. El primer punto en la red se ubicó en la conexión domiciliaria más próxima al tanque o pozo, seguida de una conexión domiciliar a una distancia intermedia y terminando en la conexión domiciliar más alejada del tanque o de la fuente del acueducto.

Los datos analizados fueron los del nivel primero N1: que corresponde al programa de control básico, junto con la inspección sanitaria, para evaluar la operación y el mantenimiento en la fuente, el almacenamiento y la distribución del agua potable. Los parámetros en este nivel son: coliformes termotolerantes (fecales), Escherichia coli, color aparente, turbiedad, olor, sabor, temperatura, $\mathrm{pH}$, conductividad, y cloro residual libre o combinado (Presidencia de la República, Ministerio de Salud, 2015), y se utilizaron los ensayos establecidos en el Standard Methods for the Examination of Water and Wastewater (American Public Health Association, American Water Works Association, Water Pollution Control Federation \& Water Environment Federation, 2017).

Además, con el fin de contribuir con la gestión de riesgos de los acueductos, se elaboraron mapas de la infraestructura y vulnerabilidad para algunas de las Asadas. Los mismos fueron realizados por estudiantes pasantes del Instituto ENGEES de Francia. Con el fin de identificar necesidades de las Asadas, se realizaron talleres participativos con miembros de las Juntas Directivas, además se aplicaron diferentes instrumentos; la construcción del árbol del problema y el análisis FODA, además de procesos de construcción de acuerdos, agendas de seguimiento y mapeo de actores sociales.

\section{Proceso de sistematización}

Los procesos de sistematización se realizan buscando el objetivo de transferir el conocimiento y compartir la información, enseñar sobre la experiencia práctica vivida, la relación con diferentes sujetos, con quienes se interactúa mediante un proceso ordenado y consciente (por lo tanto, metódico) de descubrimiento y explicación de los procesos y productos de conocimiento presentes en la práctica (Barnechea, 1994).

Los procesos de sistematización son sumamente valiosos para aprender sobre la experiencia vivida por parte de las personas proyectistas, lo cual ayuda a alimentar la toma de decisiones a futuro. En este caso, se desarrolló un proceso de sistematización basado en el "Manual para sistematizar experiencias de 
URL: https://www.revistas.una.ac.cr/index.php/dialogo/index

CORREO ELECTRÓNICO: universidadendialogo@una.ac.cr

DOI: https://doi.org/10.15359/udre.10-2.1

manejo participativo en la conservación de la biodiversidad" (Brenes, 2015), con una breve adaptación para los procesos de fortalecimiento de las Asadas. La cual consistió en la utilización de catorce pasos mediante la utilización de una ruta gráfica, según se muestra en la figura 2.

Figura 2. Pasos de la ruta gráfica del manual de sistematización de experiencias

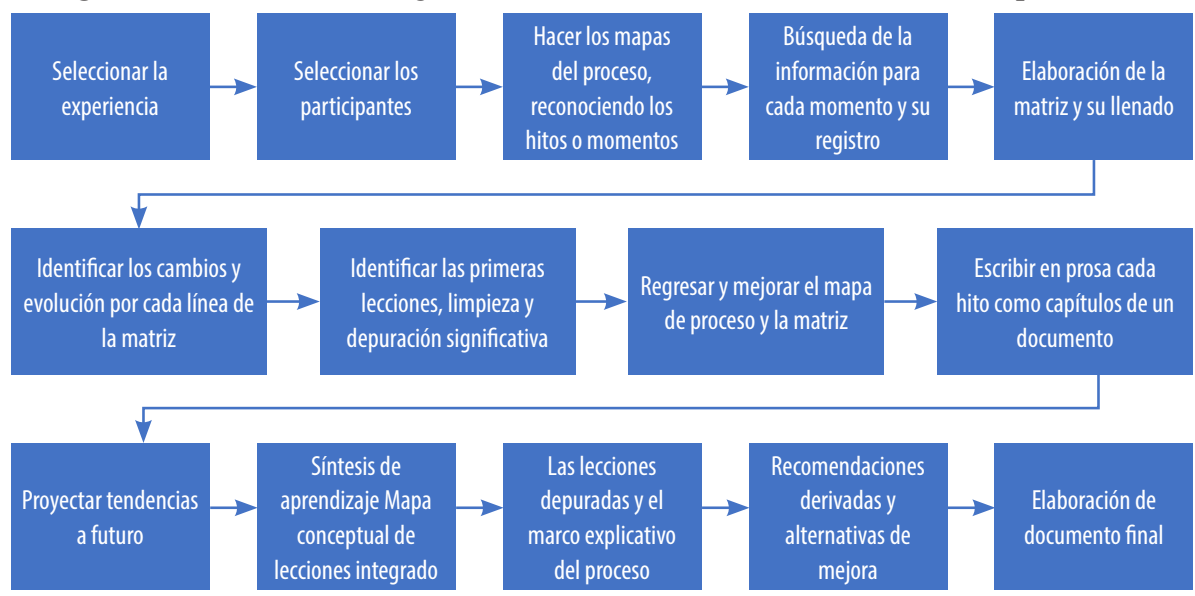

Fuente: Adaptación propia de Brenes (2015).

Por lo que a partir de esta metodología se generó la sinopsis final del proyecto, donde se rememoran y se buscan los hitos que sintetizan el aprendizaje y las experiencias aprendidas.

\section{Sinopsis en el desarrollo del proyecto}

\section{Hito 1: Presentación del proyecto a las Asadas}

Inicialmente, del total de Asadas de la región Chorotega se realizó un proceso de preselección para saber los cantones con mayor índice de vulnerabilidad del recurso hídrico. En trabajo conjunto con la Oficina de Acueductos Rurales del AyA, basado en evaluaciones previamente realizadas por esta oficina, se seleccionaron los cantones de Santa Cruz, Abangares, Nicoya y La Cruz por los problemas en escasez y salinización que presentaban, principalmente debido a la sequía de 2014 y 2015. 
Revista Universidad en Diálogo • Vol. 10, N. 2, Julio-Diciembre, 2020 • 11-33

ISSN 2215-2849 • EISSN: 2215-4752

URL: https://www.revistas.una.ac.cr/index.php/dialogo/index CORREO ELECTRÓNICO: universidadendialogo@una.ac.cr

DOI: https://doi.org/10.15359/udre.10-2.1

La convocatoria para todas las Asadas de los cantones seleccionados se realizó mediante envío de correos electrónicos y llamadas telefónicas a los miembros de las Juntas Directivas que estaban en la base de datos de la Oficina de Acueductos Rurales. En caso de no poderlos contactar, se realizaron visitas de campo a los sitios donde se verificó la veracidad de dicha información, se corrigieron los datos erróneos y se hizo nuevamente la convocatoria a todas las Asadas seleccionadas. La convocatoria inicial consistía en invitarlos a un taller donde se presentarían los objetivos y alcances del proyecto.

La agenda del taller inicial tenía como primer punto una dinámica de presentación de las personas participantes, luego se desarrolló la actividad denominada: Rompecabezas. Manejo de cuencas hidrográficas desarrollado por la Universidad Estatal a Distancia (UNED, 2013), para continuar con la presentación de cada una de las actividades que se iban a realizar durante los años del proyecto (ver figura 3). Es importante señalar que para el 2015 el taller se realizó en Santa Cruz y Abangares. En el 2016, se realizó en Nicoya y en el 2018 en La Cruz. Los talleres finalizaban con la entrega del consentimiento informado donde las Asadas se comprometían a participar en el proyecto y darle seguimiento hasta el final. Este documento debía ser aprobado por la Junta Directiva de cada Asada.

Figura 3. (A) Taller introductorio con miembros de las Asadas de Nicoya en el año 2017 y (B) la actividad de rompecabezas con miembros de las Asadas de La Cruz en el año 2019

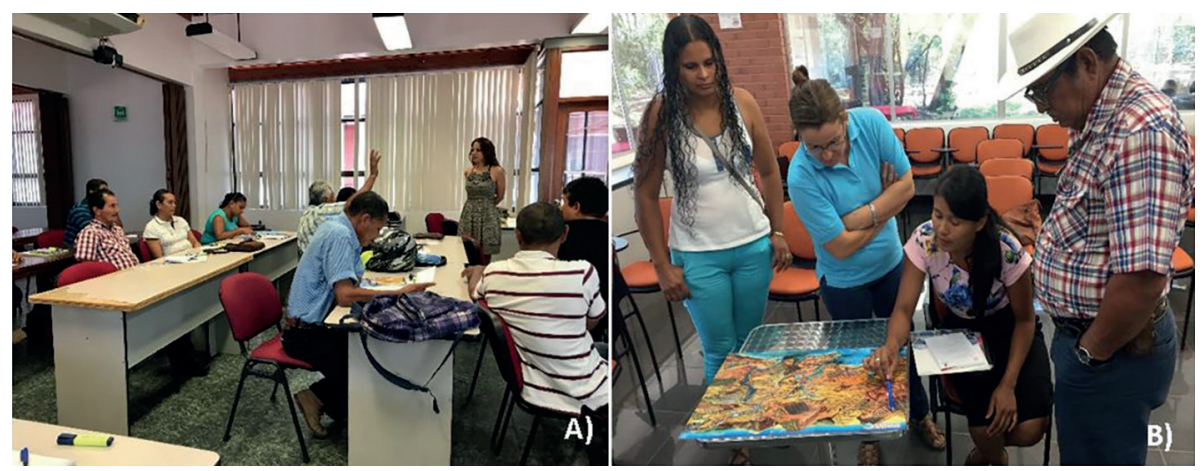

Fuente: Elaboración propia del HIDROCEC-UNA.

La convocatoria total, incluyendo todos los cantones desde el 2015 al 2018 fue de sesenta y seis Asadas, de las cuales cuarenta acudieron al taller de presentación inicial, se les preguntó a las que no asistieron las razones y estas señalaron entre las principales causas problemas organizativos dentro de las Asadas, económicos y de transporte. Esto se puede explicar debido a la 
URL: https://www.revistas.una.ac.cr/index.php/dialogo/index

CORREO ELECTRÓNICO: universidadendialogo@una.ac.cr

DOI: https://doi.org/10.15359/udre.10-2.1

disparidad en la gestión organizacional y de infraestructura de cada una de las asociaciones a nivel cantonal. Pues, hay Asadas muy consolidadas, mientras que existen otras sin infraestructura, ni Juntas Directivas, y en el extremo de los casos, tampoco cuentan con un presidente.

En el cantón de Santa Cruz, como ejemplo, la participación fue muy exitosa, debido principalmente al nivel socioorganizativo de las Asadas, ya que en este cantón existe la Unión de Asadas de la Costa (Unaguacostas). Un grupo de Asadas que se reúne cada cierto tiempo para sugerir e incentivar la mejora del recurso hídrico, lo que ayudó en gran medida a la participación de un grupo más consolidado e interesado por los procesos de capacitación (catorce Asadas participantes). Caso contrario, en Colorado de Abangares, donde no existen tejidos organizativos y hay conflictos evidentes, donde de ocho Asadas ninguna participó.

\section{Hito 2: Taller Gestión Integrada de los Recursos Hídricos}

Este primer módulo marcó un hito para el proyecto, ya que fue el que dio inicio con los procesos de capacitación; durante el mismo se plantearon importantes bases en cuanto al enfoque de la gestión del agua y se sensibilizó a las personas participantes en la importancia de la colaboración y la buena comunicación que debe existir entre los grupos de trabajo para alcanzar los objetivos (ver figura 4A). Este primer módulo se dividió en tres unidades: 1. Introducción a la Gestión Integrada de los Recursos Hídricos (GIRH); 2. Cuencas Hidrográficas y 3. Legislación.

Figura 4. (A) Ejercicios desarrollados en el módulo 2 para la comprensión de la cooperación en el alcance de un mismo objetivo y (B) cómo interpretar mapas cartográficos; realizados en el taller de capacitación con Asadas de La Cruz.
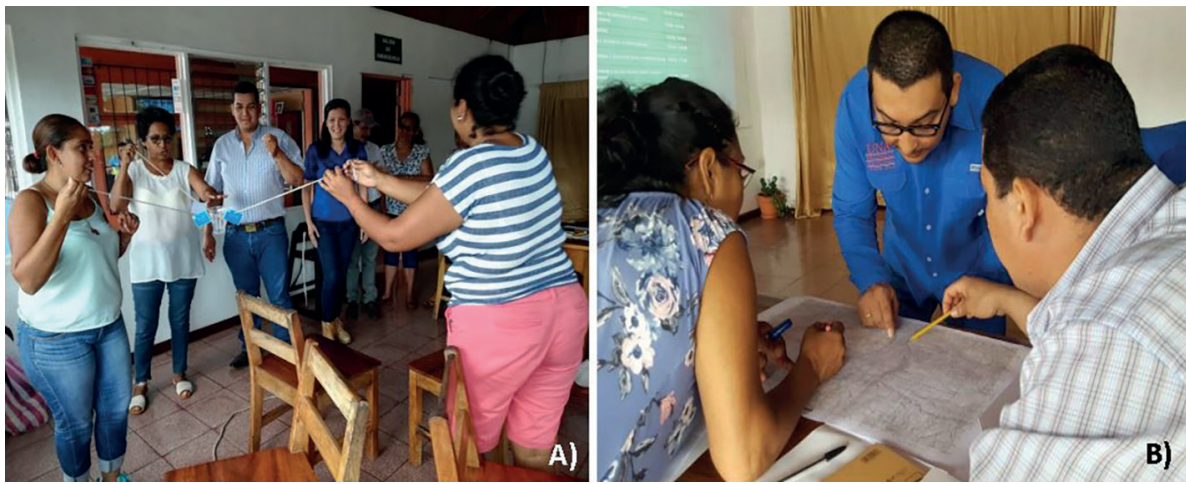

Fuente: Elaboración propia del HIDROCEC-UNA. 
La primera unidad inició con una dinámica llamada "Cuadrados rotos"; el objetivo fue analizar algunos aspectos de cooperación en la solución de problemas de grupo, sensibilizando a las personas participantes acerca de las conductas que obstaculizan o que contribuyen a la solución de problemas en un grupo. El tema de cooperación en la GIRH es fundamental, a partir de la discusión y reflexión de la actividad se empiezan a introducir los principales conceptos para entender la GIRH.

En la unidad 2 se explican de manera interactiva los conceptos de cuenca hidrográfica y cómo de manera sencilla se pueden leer mapas cartográficos (ver figura 4B). Se discute la importancia del enfoque de cuenca en el marco de la GIRH. Mientras que para la unidad 3 se realizó primero una charla explicativa con la reglamentación básica que debe conocer toda Asada en su gestión. Se realiza una actividad tipo foro de discusión donde se abre el espacio para que algunos miembros de las Asadas compartan su experiencia en diferentes procesos legales en los que han estado involucrados y cómo pudieron resolverlos.

\section{Hito 3: Taller Calidad de Aguas}

Este módulo se dividió en tres unidades que fueron impartidas en tres semanas. Las mismas fueron denominadas Calidad de aguas para consumo humano, Potabilidad y Sistemas de tratamiento de aguas residuales, respectivamente. Cada una de las unidades se impartió en aproximadamente cuatro horas, en las que se realizó una charla magistral intercalada con actividades que permitieron enriquecer el conocimiento adquirido previamente, para posteriormente hacer una discusión de resultados obtenidos.

La temática abordada en Calidad de aguas para consumo humano exhorta a los participantes del taller a adquirir conocimiento relacionado con la interpretación de datos de análisis físicos, químicos y microbiológicos de las aguas. Se realizaron ejercicios de análisis de $\mathrm{pH}$, cloro libre y total, turbiedad, entre otros. Además de un juego de concientización sobre la cantidad de agua existente en el planeta (ver figura 5).

La realización de esta primera unidad permitió la ejecución de una tarea, en la que se les solicitó a las personas participantes que realizaran una interpretación del último informe de resultados obtenido por la Asada en años anteriores. Este documento fue entregado en grupos, seleccionados según cada una de las Asadas. Se obtuvieron resultados satisfactorios, puesto que cada grupo logró entregar un documento con la interpretación correspondiente. Además, 
URL: https://www.revistas.una.ac.cr/index.php/dialogo/index

CORREO ELECTRÓNICO: universidadendialogo@una.ac.cr

DOI: https://doi.org/10.15359/udre.10-2.1

durante el proceso de sistematización y generación de los hitos, este fue uno de los talleres que tuvo la mayor evaluación positiva y es la actividad que más recuerdan las Asadas participantes del taller, por la importancia que implica conocer los parámetros que se están evaluando y los valores aceptables.

Figura 5. Ejercicio para la comprensión de los procesos de calidad de agua realizados en talleres de capacitación con Asadas de Santa Cruz (A), Nicoya (B) y La Cruz (C)

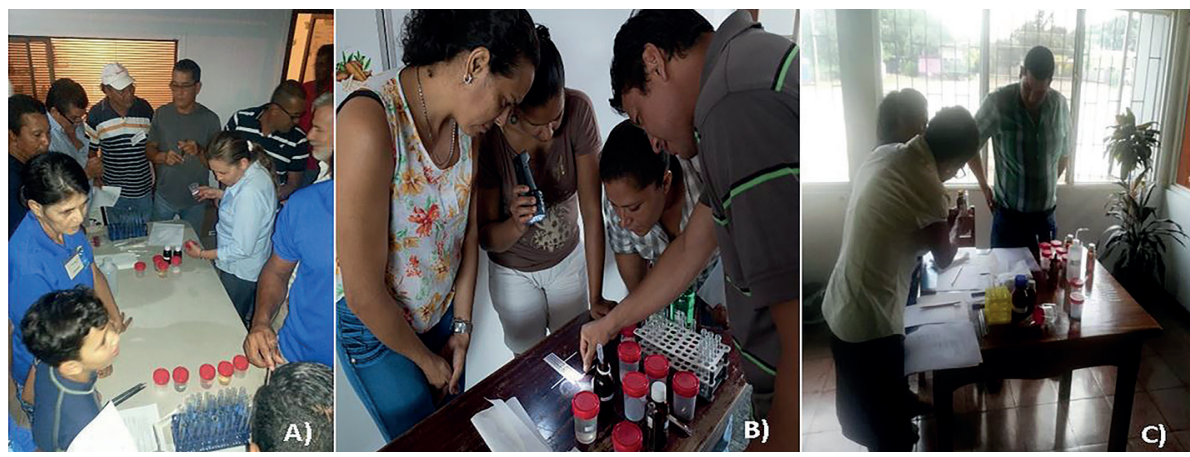

Fuente: Elaboración propia del HIDROCEC-UNA

\section{Hito 4: Medición, Ahorro y Uso Eficiente del Agua}

El desarrollo de esta actividad se dio mediante la implementación de una jornada de capacitación de cuatro horas en las que se desarrolló tanto la parte teórica, mediante charla magistral, como la discusión de los conceptos. Las temáticas abordadas incluían los principales factores que llevan a una comunidad o al individuo a consumir más agua. Se expusieron los determinantes climáticos, culturales, sociales y económicos que afectan la cantidad de agua, la comparación de estos factores con otras áreas del país y cómo influían en los recibos de pago y en la consciencia de cada una de las personas usuarias.

Lo anterior con el fin de discutir cómo las Asadas pueden intervenir para que la comunidad o las personas usuarias hagan un uso racional del recurso. La pregunta generadora llevó a un proceso de discusión sobre la realidad de cada una de las comunidades y se pudo establecer que todas las medidas están encasilladas en dos intervenciones. La primera son las estructurales, que están relacionadas con la infraestructura. El mejoramiento en las tuberías, la eliminación de fugas, el sellado de tanques y el cambio de losas sanitarias de las casas de habitación donde se identificó que existen las mayores pérdidas por fugas de agua. Luego, se analizaron algunas de las metodologías tecnológicas 
Revista Universidad en Diálogo • Vol. 10, N. 2, Julio-Diciembre, 2020 • 11-33

ISSN 2215-2849 • EISSN: 2215-4752

URL: https://www.revistas.una.ac.cr/index.php/dialogo/index CorReo eleCtrónico: universidadendialogo@una.ac.cr

DOI: https://doi.org/10.15359/udre.10-2.1

existentes para eliminar los desperdicios de agua en la parte estructural, como el cálculo de agua no contabilizada ANC y la determinación de fugas no visibles.

La segunda intervención es la social, que está determinada por el comportamiento de nosotros como seres humanos y el uso que se le da al agua por costumbre, recreación, negocio o comportamiento adquirido. Es aquí donde las campañas de concientización se hacen importantes y generan un efecto en la población. Entre los logros adquiridos se planificó desarrollar una jornada de siembra de árboles con los entes educativos de las comunidades, tanto escuelas como colegios, para involucrar a la comunidad en estos procesos. Además, se enviaron a confeccionar camisetas alusivas a la actividad para que cada una de las personas participantes en la jornada se sintiera identificada tanto ese día como en el futuro.

\section{Hito 5: Mapas de infraestructura y vulnerabilidad}

El proceso de mapeo de las Asadas consiste en visitas a campo a cada una, en las que se realiza el levantamiento de la información geográfica de los principales puntos del acueducto y su red de distribución. También se toman los sitios más representativos de cada comunidad, como, por ejemplo: iglesia, escuela, instituciones públicas y otros. Cada visita fue coordinada para poder contar con el acompañamiento de al menos un miembro de la Asada que conociera a detalle dónde se encuentra cada uno de los componentes de esta. Este trabajo se realizaba con el apoyo de estudiantes de la universidad de Engees (École Nationale du Génie de l'eau et de l'environnement de Strasbourg) en Francia. Los cuales colaboraban con pasantías para conocer sobre diferentes temáticas ambientales en Costa Rica y colaborar con el Hidrocec.

Cada recorrido consistía en la toma de la ubicación geográfica de los sitios por medio de un Sistema de Posicionamiento Global (GPS, por sus siglas en inglés), mediante el cual se obtienen los datos de las coordenadas geográficas del sitio, su elevación y el margen de error que se podría obtener por medio del posicionamiento del dispositivo. El error promedio de la ubicación del aparato GPS ronda un rango de dos a diez metros, esto según la cobertura que exista alrededor, obteniendo valores más altos en las zonas boscosas y mucho menor rango en espacios abiertos. Los modelos de GPS con los que se contó para el levantamiento fueron el GARMIN Montana 78 y el MAP 60Cx.

Los principales elementos que se tomaban con las coordenadas eran: nacientes, pozos, tanques de almacenamiento, llaves de aforo, quiebragradientes, oficinas administrativas, disminuciones en el ancho de tuberías, hidrantes y algún otro 
URL: https://www.revistas.una.ac.cr/index.php/dialogo/index

CORREO ELECTRÓNICO: universidadendialogo@una.ac.cr

DOI: https://doi.org/10.15359/udre.10-2.1

elemento significativo del sistema de distribución y abastecimiento de las Asadas. Otra medición que se realizaba era la de la red de distribución de las tuberías de todo el sistema de la Asada, la cual consistía en un recorrido por toda la red. Este proceso se realizaba por medio del levantamiento de un track, que consiste en que el GPS realiza la ruta por la cual el instrumento es desplazado en el terreno. Otra opción, en el caso de tuberías con distribución uniforme, es tomar el punto de inicio y luego el punto de fin de las tuberías, pero esto solo funciona en sistemas con distribución lineal y con distancias considerables para no provocar errores en la toma de los datos.

Con los datos de campo, se procedió a descargarlos en un ordenador y por medio de programas informáticos de sistemas de información geográfica se ordenaron y generaron los mapas de la infraestructura de las Asadas. Además, se utilizó información de bases de datos en línea o atlas geográficos para obtener información adicional, como ríos, caminos, modelos de elevación digital y otros, que permitían complementar la generación de cada mapa, como se observa en la figura 6.

Figura 6. Procesos desarrollados en el hito 6 para la generación de los mapas de infraestructura y vulnerabilidad. (A) Recorrido para colecta de datos con GPS; (B) entrega del mapa de riesgos a una Asada de La Cruz; y (C) generación del mapa de infraestructura y vulnerabilidad confeccionado y entregado a una Asada de Santa Cruz

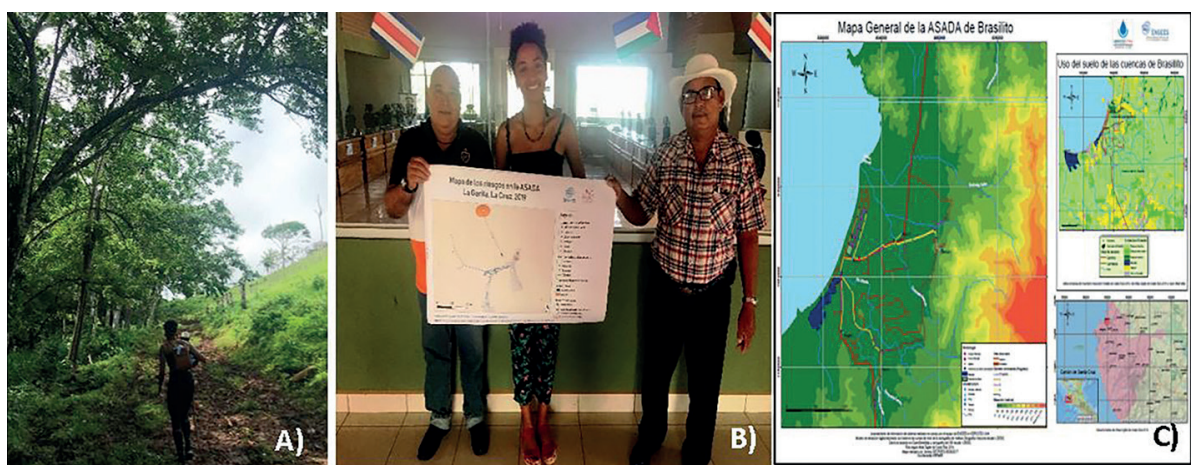

Fuente: Elaboración propia del HIDROCEC-UNA.

Una vez que se tenían listos los mapas, estos eran impresos y posteriormente se coordinaba con la Asada para la entrega del mismo, donde se les explicaba su contenido y se aclaraban las dudas que tuvieran. 
La vulnerabilidad de los sistemas de las Asadas se determinó a través de observaciones en el campo, también por medio de los talleres participativos con los miembros de las Asadas y la información obtenida del cuestionario unificado. Entre los parámetros identificados que más influyen en la vulnerabilidad de las Asadas están las fugas y su detección, la longevidad de los sistemas de tuberías, las posibilidades de vandalismo, los incendios en zonas donde se encuentre el sistema de distribución y otros. Una de las formas de identificar las vulnerabilidades fue por medio del mapa con la distribución de las Asadas, con el cual los miembros de esta ubicaban los sitios donde consideraban ellos que estaban presentes las afectaciones o los lugares donde podría ocurrir alguna afectación.

\section{Hito 6: Gestión de riesgo ante el cambio climático y los desastres naturales}

Una vez identificados los puntos más vulnerables de cada una de las comunidades, se planteaban alternativas para combatir esas afectaciones de manera conjunta y participativa entre el equipo de trabajo y los miembros de la Asada y la comunidad que participan en los talleres, además del apoyo de los estudiantes pasantes en los procesos. Muchas de las soluciones para la atención de las diferentes problemáticas quedaban sin atención por cuestiones financieras, para lo cual a lo interno de cada sistema comunal se planteaban las posibles soluciones para atender los puntos más críticos por orden de prioridades.

Por las condiciones topográficas de las zonas, existían pocas posibilidades de que se presentaran afectaciones por deslizamientos, pero en las zonas donde sí podía ocurrir este riesgo los miembros de la asociación proponían acciones como la limpieza de zonas con taludes, el establecimiento de gradientes o los movimientos de tierra para controlar la erosión, entre otras. Un elemento que está presente en la zona es el riesgo por incendios, principalmente en la época seca, que puede afectar las zonas boscosas donde se encuentran los acuíferos, por lo que una de las medidas más recurrentes en las asociaciones comunales es la alerta temprana para evitar la propagación de estos en las regiones. La protección de las nacientes, acuíferos y zonas de recarga, por lo que la reforestación de las áreas de amortiguamiento y protección de los cuerpos de agua es un factor que las Asadas de la región protegen y mantienen como medida para garantizar la recarga y retención del agua en las fuentes, además de que es una de las principales medidas de mitigación de los efectos del cambio climático. 
URL: https://www.revistas.una.ac.cr/index.php/dialogo/index

CORREO ELECTRÓNICO: universidadendialogo@una.ac.cr

DOI: https://doi.org/10.15359/udre.10-2.1

Ante las condiciones de sequía presentes en la zona, una de las medidas adoptadas por los gestores del agua es el racionamiento en los periodos secos, para así garantizar el constante abastecimiento de líquido a toda la población. Estos racionamientos cesan en el periodo lluvioso cuando los acuíferos recuperan sus caudales y los niveles de recarga, esta situación varía en cada sitio, ya que las que se encuentran en las regiones de mayor altitud o con menor población abastecida casi no cuentan con el problema del desabastecimiento por escasez de agua durante todo el año. El problema de disponibilidad de agua ha generado problemas entre la población, con acusaciones que van desde la distribución desigual del agua entre los beneficiados hasta problemas entre comunidades aledañas que no permiten el abastecimiento a otras localidades, por lo que los procesos de capacitación y de fortalecimiento de capacidades a estos grupos es una herramienta fundamental para el adecuado funcionamiento de estas pequeñas organizaciones.

\section{Hito 7: Taller Llegando a Acuerdos}

Este taller se considera un hito porque concluyó con los módulos de capacitaciones. En este módulo se desarrollaron los principales conceptos básicos de negociación. Se realizó una dinámica con los participantes para aplicar de manera práctica lo aprendido. Esta actividad fue muy enriquecedora porque ellos representaron situaciones por las que han tenido que pasar o estaban lidiando en ese momento.

Se realizó una reflexión final de las representaciones que realizaron con el objetivo de hacer una retroalimentación de las mejores herramientas que se pueden aplicar a la hora de lidiar con situaciones adversas a lo interno de la Asada y a lo externo, procurando la resolución de las diferencias sin tener que llegar al conflicto. En caso de llegar a un conflicto determinado, se les brindaron diferentes herramientas para poder abordar la situación de la mejor manera.

\section{Hito 8: Realización de encuestas y análisis N1 a todas las Asadas participantes}

La realización de las encuestas se llevó a cabo durante las giras de muestreo en los sistemas de agua potable de las Asadas. El equipo de investigadores que visitó la Asada durante la gira se dividió en dos: los que realizaban el muestreo de agua potable y los que aplicaban la encuesta. 
Revista Universidad en Diálogo • Vol. 10, N. 2, Julio-Diciembre, 2020 • 11-33

ISSN 2215-2849 • EISSN: 2215-4752

URL: https://www.revistas.una.ac.cr/index.php/dialogo/index CORREO ELECTRÓNICO: universidadendialogo@una.ac.cr

DOI: https://doi.org/10.15359/udre.10-2.1

El formulario unificado es utilizado por la Subgerencia Gestión de Sistemas Comunales del AyA para evaluar a las Asadas del país en los siguientes componentes: gestión administrativa y financiera, gestión comercial, gestión comunal, gestión del recurso hídrico y gestión de los sistemas de agua. Según la puntuación que la Asada obtenga en cada uno de los componentes, se realiza una ponderación y se clasifican las Asadas con las siguientes categorías: a) entre 80-100 puntos: consolidada; b) 60-79 puntos: desarrollo alto; c) 40-59 puntos: desarrollo bajo; d) menos de 40 puntos: débil.

La aplicación de la encuesta se hizo principalmente a la persona que figuraba como administradora o secretaria de la Asada, y en otros casos, al mismo fontanero (ver figura 7). Durante la actividad se les explicó inicialmente que el objetivo de la encuesta era funcionar como una herramienta para recolectar datos y así poder conocer la condición de la Asada desde varias brechas en el funcionamiento, como son la gestión administrativa y financiera, la gestión de sistemas de agua potable, la gestión del recurso hídrico, la gestión comunal y la gestión comercial. También se les señaló que algunas preguntas podrían contestarlas únicamente si así lo deseaban.

Figura 7. Actividades desarrolladas en el hito 9. (A) Aplicación del "Formulario unificado" y (B) colecta de muestras de agua para realizar análisis N1; ambas en distintas Asadas de La Cruz

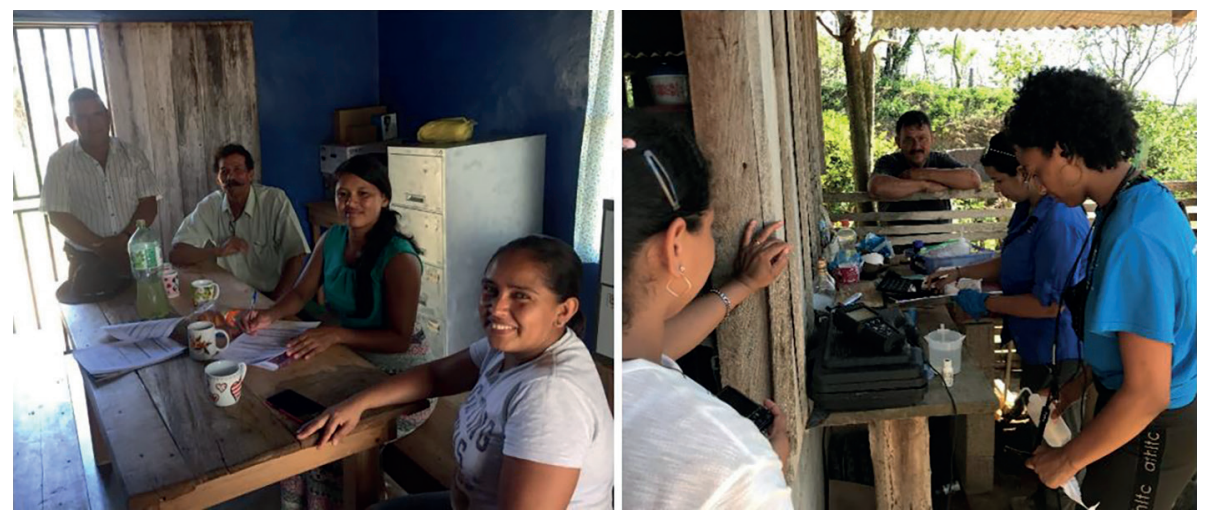

Fuente: Elaboración propia del HIDROCEC-UNA.

La herramienta aplicada resultó ser bastante larga, con preguntas repetidas y, en algunos casos, preguntas ambiguas. Esta condición generó principalmente atrasos a la hora de ejecutarla, ya que las encuestas deberían ser más puntuales. Algunas personas que contestaron la encuesta no comprendían la pregunta, entonces posiblemente contestaron erróneamente. Aun así, la herramienta fue 
URL: https://www.revistas.una.ac.cr/index.php/dialogo/index

CORREO ELECTRÓNICO: universidadendialogo@una.ac.cr

DOI: https://doi.org/10.15359/udre.10-2.1

útil porque su aplicación permitió conocer información, dar aportes a través de recomendaciones y socializar con las personas, con lo que se logró conocer los problemas que enfrentan como asociación. Situaciones tales como la falta de una infraestructura donde se realicen las gestiones de la Asada y una bodega con suministros para el mantenimiento básico del sistema.

La encuesta deja ver los faltantes e incumplimientos que tienen las Asadas, las cuales en $90 \%$ de los casos resultan con un desarrollo débil y bajo. Se observa también la división de género con respecto a las funciones que poseen las personas contratadas por la Asada; las mujeres en puestos administrativos o de limpieza, y los hombres principalmente como fontaneros.

Las Asadas tienen mucho desconocimiento con respecto a trámites administrativos, disponibilidad de agua potable (calidad y cantidad) y protección de fuentes. Además, no parecen estar muy interesadas en procedimientos burocráticos que exige el AyA, por lo que la prioridad en todo momento es que haya agua disponible. Las personas que dirigen las Asadas se distinguen por su interés en trabajar para el pueblo, pero necesitan mucho apoyo y capacitación por parte de instituciones públicas y de la academia. También se requiere colaboración por parte de la población beneficiada, para proteger el recurso de la contaminación y del desperdicio.

Durante los muestreos también se realizó la toma de muestra de agua para el análisis N1 en los pozos, los tanques y los tres puntos en la red, según el Reglamento para la Calidad del Agua Potable ( No $^{\circ} 38924-\mathrm{S}$ ). Las visitas permitieron hacer observaciones y recomendaciones sobre la infraestructura, de manera que si se identificaba alguna situación que podría poner en riesgo la calidad de las aguas de consumo se le indicaba a la persona representante de la Asada. Además, como se realiza la medición en campo de algunos parámetros ( $\mathrm{pH}$, conductividad, temperatura, cloro) en presencia del miembro de la Asada, se aprovecha para explicar ciertas condiciones que se están dando y que podrían afectar la calidad del agua.

Según lo observado en los análisis N1, las Asadas estudiadas no realizan cloración en el pozo, por lo que sus niveles de cloro libre son no detectables. La cloración se lleva a cabo en el tanque, en su mayoría a través de un sistema de pastillas. Sin embargo, del total de las Asadas estudiadas, únicamente el 20 $\%$ cumplió con el rango de valores óptimo (0.3-06 mg/l cloro residual libre), según el Reglamento para la Calidad del Agua Potable (No 38924-S-2015). Esta situación tiene una importante relación con los datos obtenidos con los análisis microbiológicos, donde se observa que, durante los muestreos, las Asadas revelan contenidos importantes de coliformes totales durante la época lluviosa. 
La identificación de microorganismos en las muestras de aguas evaluadas, tanto en el pozo como en el tanque y en la red de distribución, implica un riesgo de vulnerabilidad, pues la Asada que presentó mayor número de coliformes fecales y $E$. coli no utilizaba clorador para el tratamiento del agua de consumo. La contaminación de aguas de consumo con materia fecal de ganado vacuno, así como con aguas negras o materia fecal humana, se ha asociado con brotes de diarrea. Sin embargo, es importante resaltar que no solamente bacterias pueden ingresar al sistema, sino también los llamados ooquistes de protozoario, los cuales son causa de diarrea infantil en Costa Rica. Por lo que es sumamente importante fortalecer las capacidades de las Asadas en este sentido.

\section{Hito 9: Entrega de camisetas y de árboles para jornada de reforestación}

La entrega de camisetas y de árboles como jornada de reforestación fue una actividad con muchos beneficios. Las personas que participaron, desde niños y niñas hasta personas de la tercera edad, se sintieron motivadas y se mostraron agradecidas. En algunas Asadas, las personas a las que se les entregaron camisetas, las usaron el día de la siembra de los árboles. Las camisetas fueron entregadas con diferentes tamaños, así que todas las personas participantes pudieron utilizar la suya.

Los árboles entregados eran de diferentes especies, entre estos frutales y maderables. Los árboles frutales otorgan alimento y hábitat a diferentes animales, y estos a su vez contribuyen con la germinación y polinización para el crecimiento del bosque. Sin embargo, en algunas comunidades donde no hubo acompañamiento, los árboles se sembraron a las orillas de los parques públicos.

Se sabe que una actividad como esta trae beneficios ecológicos; la siembra de los árboles en zonas de recarga de agua subterránea de la cuenca o en zonas circundantes a las instalaciones de los pozos protege este recurso de la contaminación, contribuye con la infiltración y evita la erosión de las tierras. Sin embargo, en algunas Asadas los árboles fueron entregados en época seca, por lo que muchos de estos, después de la siembra, fueron descuidados, ya que ninguna o muy pocas personas se hicieron responsables del riego. Por lo que, como recomendación para próximas actividades similares, se debe llevar un monitoreo adecuado después de las siembras, para estimar el porcentaje de árboles que se sembró y el porcentaje que sobrevivió. 
URL: https://www.revistas.una.ac.cr/index.php/dialogo/index

CORREO ELECTRÓNICO: universidadendialogo@una.ac.cr

DOI: https://doi.org/10.15359/udre.10-2.1

\section{Hito 10: Sistematización y retroalimentación}

El proceso de la recuperación de la experiencia vivida durante el proceso de ejecución del proyecto permite que el equipo de trabajo pueda identificar las fortalezas del proyecto y las diferentes acciones de mejora para llevar a cabo una adecuada gestión de los recursos, así como las acciones de mejora para poder replicar la experiencia desarrollada en otras poblaciones vulnerables. Esta recuperación histórica del proceso se ha realizado de manera participativa entre el equipo ejecutor y los diferentes participantes de la experiencia, la metodología empleada consistió en la reconstrucción histórica por medio de la consulta de la documentación existente, como listas de asistencia de las sesiones de trabajo, minutas, material fotográfico, consultas a los actores del proceso y otras. Al ser un proceso participativo, permite que cada actor involucrado arme desde su punto de vista una visión globalizada del trabajo desarrollado, para así poder formar un criterio conjunto de las acciones ejecutadas.

Uno de los elementos que fortalece este proceso de sistematización de la experiencia es la reconstrucción activa con la población de las Asadas, desde el punto de vista de ellos como beneficiados del proyecto. En ocasiones existe cierta limitación en la manera de responder sobre el proceso, ya que la mayoría se enfoca en las buenas experiencias vividas. Al generar un ambiente de confianza, se puede indagar en los aspectos "negativos" que ellos no quieren abordar, porque les genera pena o simplemente sienten que se podrían evitar un problema. Pero obtener información de esos elementos que ellos consideran que el equipo puede mejorar permite que ambas partes se vean beneficiadas, ya que ellos externalizan esos elementos, y por parte del equipo ejecutor se pueden dar las debidas explicaciones del caso y tomar consideraciones para futuras acciones a desarrollar.

\section{Conclusiones y recomendaciones}

- La selección de las Asadas realizada permitió trabajar con Asadas con capacidades disminuidas en recurso y en administración. Esto fue beneficioso, pues se pudieron fortalecer sus acueductos en aspectos importantes de manejo de las Asadas, gestión de riesgo y manejo administrativo, que fueron evidentes al final del proceso. Esto al comparar los estados antes y después del desarrollo del proyecto.

- Se evidencia una diferencia marcada en las condiciones económicas y administrativas de los distintos acueductos en los que se trabajó. 
Estas diferencias no son únicamente en la infraestructura y los tamaños de las Asadas, sino también en las capacidades de organización de sus miembros y las problemáticas que enfrentan. Estas diferencias hicieron que unas Asadas fueron más receptivas y colaboradoras que otras en la creación de los procesos y las acciones.

- Durante el proceso se aplicó a todas las Asadas el "Formulario unificado" de información sobre prestadores de los servicios de acueductos, saneamiento y fuentes de las instituciones del Estado. Esta es una herramienta de mucha importancia para conocer el funcionamiento interno de cada Asada y darle una valoración para su fortalecimiento, sin embargo, la aplicación del instrumento en algunos casos generó desconfianza, pues sintieron que era un proceso de evaluación, además de que el instrumento resulta largo.

- Las Asadas de Santa Cruz parecen tener un tejido organizativo más consolidado que las Asadas de los cantones de Nicoya y Abangares. Esto principalmente debido al problema que los aqueja y a la participación de varias instituciones en los procesos de búsqueda de soluciones, tal es el caso de la comisión Conimboco. Donde intervienen otras instituciones, como Senara, Dirección de Agua, PNUD, CFIA, entre otras.

\section{Referencias bibliográficas}

Acueductos y Alcantarillados (2010). Calidad del agua en sus diferentes usos en Guanacaste. San José, Costa Rica: AyA.

Acueductos y Alcantarillados (2015). Ventajas de la micromedición de un acueducto. San José, Costa Rica: AyA.

Acueductos y Alcantarillados (octubre, 2016). Proyecto AyA 2010-2017. Recuperado de https://www.aya.go.cr/proyectos/SitePages/Detalle\%20 del $\% 20$ proyecto.aspx? spidProyecto $=22$

Acueductos y Alcantarillados (2018). Formulario unificado. Recuperado de https://www.aya.go.cr/ASADAS/documentacionAsadas/ FORMULARIO\%20UNIFICADO.pdf 
American Public Health Association, American Water Works Association, Water Pollution Control Federation \& Water Environment Federation (2017). Standard methods for the examination of water and wastewater (Edición 23). USA: American Public Health Association.

Barnechea, M. (1994). La sistematización como producción de conocimientos. Memoria del Seminario de Intercambio y Debate sobre Sistematización. Lima, Perú.

Brenes, C. (2015). Manual para sistematizar experiencias de manejo participativo en la conservación de la biodiversidad. Heredia: MAPCOBIO-SINAC-JICA.

Estado de la Nación (2007). Recurso Aguas Superficiales y Subterráneas con énfasis en las principales cuencas hidrográficas. San José, Costa Rica.

Organización Mundial de la Salud (2004). Guías para la calidad del agua potable. Volumen 1.

Presidencia de la República, Ministerio de Salud. (2015). Reglamento para la Calidad del Agua Potable No 38924-S. La Gaceta.

Universidad Estatal a Distancia (2013). Proyecto Guía para el uso del rompecabezas Manejo de Cuencas Hidrográficas. Costa Rica: UNED. Recuperado de https://www.uned.ac.cr/extension/extension-en-accion/ noticias/888-proyecto-guia-para-el-uso-del-rompecabezas-manejo-decuencas-hidrograficas

Van Noorloos, F. (2013). ¿Un lugar en el sol para quién? El turismo residencial y sus consecuencias para el desarrollo equitativo y sostenible en Guanacaste, Costa Rica. AlbaSud. 